\title{
Correlation of SOX4, SOX17, VE-cadherin and vasculogenic mimicry with the development and prognosis of esophageal squamous cell carcinoma
}

\section{Yanzi Qin ( $\sim 18725525611 @ 163 . c o m)$}

First Affiliated Hospital of Bengbu Medical College https://orcid.org/0000-0003-4241-9638

\section{Wenjun Zhao}

The Third People's Hospital of Bengbu

\section{Zhaogeng Cai}

First Affiliated Hospital of Bengbu Medical College Department of Rheumatology

\section{Qi Wang}

First Affiliated Hospital of Bengbu Medical College

\section{Jin Gao}

First Affiliated Hospital of Bengbu Medical College Department of Rheumatology

\section{Ningning Yang}

First Affiliated Hospital of Bengbu Medical College Department of Rheumatology

Hongfei Ci

First Affiliated Hospital of Bengbu Medical College Department of Rheumatology

\section{Zhenzhong Feng}

First Affiliated Hospital of Bengbu Medical College Department of Rheumatology

\section{Li Ma}

First Affiliated Hospital of Bengbu Medical College Department of Rheumatology

\section{Original Article - Cancer Research}

Keywords: esophageal cancer, squamous cell carcinoma, SOX17, SOX4, VE-cadherin, vasculogenic mimicry

Posted Date: February 3rd, 2021

DOl: https://doi.org/10.21203/rs.3.rs-166570/v1

License: (c) (1) This work is licensed under a Creative Commons Attribution 4.0 International License. Read Full License 


\section{Abstract}

Background: We previously reported high SOX4 expression in esophageal squamous cell carcinoma (ESCC), which participates in the mechanism of vasculogenic mimicry (VM) by mediating the epithelialmesenchymal transition (EMT) mechanism. In this study, the relationships between SOX4, SOX17, vascular endothelial (VE)-cadherin and VM were analyzed to explore the mechanisms of the occurrence and development of ESCC.

Methods: SOX4, SOX17, and VE-cadherin expression as well as VM in 210 ESCC tissues and 60 normal esophageal mucosal tissues were determined by immunohistochemistry (IHC), and correlations with clinicopathological parameters were explored. The invasion, migration, and proliferation of EC9706 and Eca109 cells were determined after silencing of VE-cadherin with siRNA interference technology. SOX4, SOX17, and VE-cadherin protein and mRNA expression were quantified by Western blotting and qRT-PCR analyses, respectively.

Results: Low SOX17 expression, high SOX4 expression, and high VE-cadherin expression were observed in ESCC tissues and were significantly correlated with tumor size, lymph node metastasis (LNM), depth of invasion, and pathological tumor node metastasis (pTNM) stage. They also were independent poor prognostic factors in ESCC patients. After VE-cadherin silencing, the invasion, migration, and proliferation of EC9706 and Eca109 cells in vitro were decreased, while SOX17 protein and mRNA levels were increased and SOX4 protein and mRNA levels were decreased.

Conclusions: SOX17, SOX4, and VE-cadherin are involved in the development of ESCC. Low expression of SOX17 and high expression of SOX4 may promote VM in ESCC by enhancing VE-cadherin transcription.

\section{Background}

The incidence of esophageal cancer $(E C)$ has been steadily increasing year after year, making EC the sixth most common cause of cancer-related death in the world [1]. The incidence and mortality of EC in China continue to remain high [2] with $90 \%$ of cases being esophageal squamous cell carcinoma (ESCC) [3]. Despite being a common cancer, the process of tumorigenesis in EC remains unclear.

The growth of a solid tumor depends upon its vascularity. Anti-angiogenic therapies are designed to target vascular endothelial cells and prevent formation of tumor blood vessels [4]. Vasculogenic mimicry (VM) is a recently identified tumor micro-circulation model that is independent of the organism's endothelial cells, and its growth model is completely different from the classical tumor vascular growth model [5]. Vascular endothelial cadherin (VE-cadherin) is a specific transmembrane adhesion protein found on the surface of vascular endothelial cells. It maintains the integrity of vessels and promotes adhesion between adjacent endothelial cells [6,7]. Recent studies have shown that overexpression of VEcadherin may be an important regulatory mechanism for VM $[8,9]$. 
In the early 1990s, the discovery of the sex-determining region on Y (SRY) led to the identification of the SRY-related box (SOX) transcription factors $[10,11]$. These factors often have pleiotropic functions that can lead to the activation of alternate transcriptional programs [12-14]. SOX17, first cloned from cDNA libraries of mouse testicular tissue, was found to have a stage-specific function in spermatogenesis [15]. Later, SOX17 was found to have anti-proliferative effects in endometrial cancer by suppressing the transcription of Notch effector MAML3, a co-activator of $\beta$-catenin [16]. In a colonic carcinoma model, SOX17 antagonizes $\beta$-catenin signaling by redirecting $\beta$-catenin away from WNT target genes and by depleting its protein levels via the GSK3-independent promotion of its proteasomal degradation [17]. On structure function analysis, SOX17 was found to be inactivated in colon cancer [18], lung cancer [19], and hepatocellular carcinoma [20].

SOX4 is a member of the SOX family that contains a highly conservative and migratory DNA-binding domain. The translated proteins are 47-kDa transcription factors involved in development [20]. Previous studies have found that SOX4 is one of the 64 "tumor characteristic" genes, and its expression is upregulated in a variety of tumor tissues, such as bladder cancer, liver cancer, prostate cancer, and acute myelogenous leukemia [21, 22]. Over-expression of SOX4 promotes the invasion and migration of cancer cells, and SOX4 binds to the p53 promoter to inhibit the pro-apoptotic effect of p53, thereby reducing the therapeutic effect of radiotherapy [23].

We previously reported high expression of SOX4 in ESCC and the involvement of VE-cadherin in VM formation. SOX4 over-expression promotes the development of epithelial-mesenchymal transition (EMT) which allows the tumor cells to remodel. Also, the tumor cells with VM structure were found to be separated from the lumen by only one layer of PAS-positive substance [24]. However, the exact mechanistic roles of SOX4 and 17 in ESCC remain unclear. Whether SOX4 and SOX17 interact together to participate in the formation of VM in ESCC is not yet known. This experimental study intended to observe the effect of VE-cadherin silencing via siRNA interference on the expression of SOX4 and SOX17 in ESCC and the corresponding impacts on the invasion and metastasis of ESCC.

\section{Materials And Methods}

In this study, tissue samples from 210 patients with ESCC and 60 patients with normal esophageal mucosa were collected from the First Affiliated Hospital of Bengbu Medical College from January 2014 to December 2015. The tissue samples of ESCC were obtained from surgical specimens. None of the patients with ESCC included in this study had received chemotherapy or radiotherapy before the surgery. Detailed clinicopathological data of the study participants are presented in Table 1. Additionally, fresh saline tissue samples of ESCC and adjacent normal tissues were collected from 10 patients between 
August 2019 and December 2019 from the First Affiliated Hospital of Bengbu Medical College. These samples were placed in liquid nitrogen immediately for later use in western blot analysis. For detection of mRNA levels, the fresh tissues were immersed in RNA store solution (TIANGEN, Beijing, China) in the ratio of $1: 10$ and stored in liquid nitrogen at $4^{\circ} \mathrm{C}$ overnight.

\section{Immunohistochemistry (IHC)}

The paraffin-embedded samples were sectioned into 3- $\mu \mathrm{m}$-thick slices. After dewaxing and debenzenization, limonic acid high-pressure antigen repair, anti-SOX17 antibody (dilution ratio 1:150, AB224637, Abcam, USA), anti-SOX4 antibody (dilution ratio 1:150, AB236557, Abcam, USA), anti-VEcadherin antibody (dilution ratio 1:200, AF6265, Affinity Biosciences, USA), and CD34 (dilution ratio 1:250, AB110643, Abcam, USA) were added, separately. 3, 3-N-DiaminobenzidineTetrahydrochloride (DAB) color was added to the treated slices.

In SOX17- and SOX4-positive cells, flaky or granular brownish yellow staining was seen in the nucleus or cytoplasm, while VE-cadherin-positive cells showed flaky or granular brownish yellow staining of the cell membrane and cytoplasm. The staining results included the proportion of positive cells and staining intensity [25]. The proportion of positive cells refers to the percentage of positive cells among the total observed cells of the same species: 0 ( $\leq 10 \%) ; 1$ point $(11 \sim 25 \%) ; 2$ points $(26 \% \sim 50 \%) ; 3$ points $(51 \% \sim 75 \%)$; and 4 points (> 75\%). Staining intensity was graded as $0,1,2$ and 3 points for no staining, light yellow, brownish yellow and tan yellow staining, respectively. The points for percentage of positive cells and staining intensity were multiplied, and the mean value was calculated to decide the staining results as follows: $0 \sim 3$ was considered negative, and $4 \sim 12$ was taken as positive.

For all CD34-stained immunohistochemical sections, DAB color development was performed, and the color development reaction was stopped by washing with flowing water for $1 \mathrm{~min}$. The cells were rinsed with water for $2 \mathrm{~min}$ and then stained with periodic acid-Schiff (PAS) for 15 30 min. The cells were again rinsed with distilled water thrice for 1 min each. VM was detected by the presence of tumor cells around the PAS-positive and CD34-negative tubes with few necrotic tumor cells and inflammatory cells infiltrating the surrounding tissues, and absence of red blood cells in the lumen of the tubes. Endothelium-lined normal vessels were identified by the presence of CD34-positive endothelial cells in their wall. 
The human ESCC cell lines EC9706 and Eca109 were grown in Dulbecco's Modified Eagle's Medium (DMEM, Hyclone, USA), supplemented with $10 \%$ fetal bovine serum (FBS, Gibco, USA) in $5 \% \mathrm{CO}_{2}$ at $37^{\circ} \mathrm{C}$. The cell lines were divided into three groups: control group, control siRNA group, and VE-cadherin siRNA group. In the control group, EC9706 and Eca109 cells were not treated. In the control siRNA group, EC9706 and Eca109 cells were infected with the empty plasmid. In the VE-cadherin siRNA group, EC9706 and Eca109 cells were infected with a lentivirus encoding precursor VE-cadherin or vector and treated with puromycin for 2 weeks to obtain stably transfected cells.

\section{siRNA transfection}

EC9706 and Eca109 cells were seeded into six-well plates and transfected with VE-cadherin siRNA and a negative control (NC, GenePharma, China) using Lipofectamine 2000 (Invitrogen, USA) according to the manufacturer's instructions. The siRNA sequence was: CCAUUGUGCAAGUCCACACAUTT (forward, 5'-3') and AUGUGGACUUGCACAAUGGTT (reverse, $5^{\prime}-3^{\prime}$ ). The cells were subjected to analysis as described in the Results section.

\section{Western blotting}

An appropriate amount (250 500 mg) of fresh tissue or properly preserved tissue was immersed in $1 \mathrm{ml}$ of strong radio-immunoprecipitation assay (RIPA) buffer containing phenylmethylsulfonyl fluoride (PMFS) was added. An electric homogenizer was used to produce the homogenate. The samples were collected after addition of lysis buffer and placed on ice for cracking for 20 30 min. Then centrifugation was performed at $12000 \mathrm{rpm}$ for $10 \mathrm{~min}$. ESCC cells were lysed in RIPA buffer with proteinase inhibitors. Protein concentrations were quantified using a BCA assay kit (Beyotime Biotechnology, China). Subsequently, the proteins were isolated by sodium dodecyl sulfate-polyacrylamide gel electrophoresis (SDS-PAGE) and transferred onto polyvinylidene fluoride (PVDF) membranes (Millipore, USA).

Membranes were incubated in $5 \%$ non-fat milk and immunoblotted with the following antibodies: antiSOX17 antibody (diluted 1:500, AB224637, Abcam, USA), anti-SOX4 antibody (diluted 1:500, AF2610, Affinity Biosciences, USA), anti-VE-cadherin antibody (diluted 1:1000, AF6265, Affinity Biosciences), and anti- $\beta$-actin antibody (Cell Signaling Technology, USA).

\section{Quantitative real-time PCR}

Trizol (Invitrogen, USA) was used to extract mRNA from ESCC tissues or cells, and the extracted mRNA samples were reversely transcribed into CDNA templates. Quantitative real-time PCR was performed using an ABI 7900 System with SYBR green (TaKaRa, China). The primers for SOX4 were as follows: AGCAAGAAGGCGAGTTAGTT (forward, 5-3') and TGACCAAGA AGCAAAATAAAA (reverse, 5'-3'). The 
primers for SOX17 were as follows: GGTTTTTGTTGCTGTTG (forward, 5'-3') and AACTTGGAAATAGGGTTTTGAC (reverse, 5'-3'). The primers for VE-cadherin were as follows: TACCAGCCAAGTTGTGA (forward, 5'-3') and GCCGTGTTATCGTGATTATCC (reverse, 5'-3'). The transcript levels were quantified by normalization to GAPDH expression (5'-CTGGGCTACACTGAGCACC, forward, 5'-3' and AAGTGGTCGTTGAGGGCAATG, reverse, 5'-3') as an internal standard.

\section{Wound healing assays}

Two cell lines were seeded overnight in six-well plates followed by transfection with VE-cadherin siRNA or NC siRNA. When cells reached greater than $90 \%$ confluency, the tip of a pipette was used to make a wound, and the detached cells were rinsed away with phosphate-buffered saline (PBS). Images of the wounds were taken at $0 \mathrm{~h}$ and $24 \mathrm{~h}$.

\section{MTT assay}

ESCC cell lines were seeded overnight into 96 -well plates at $5 \times 10^{3}$ cells per well. Subsequently, the cells were transfected with VE-cadherin siRNA for $72 \mathrm{~h}$. Cell viability was measured by MTT assay as described previously [25].

\section{Transwell migration and invasion assay}

Cell migration and invasion were evaluated by Transwell assay as previously described [26]. Briefly, transfected ESCC cells were seeded in 24-well plates with 8- $\mu$ m-pore-size chamber inserts (Corning, USA). The upper chambers were coated with Matrigel (BD Biosciences, USA) before cell seeding. After incubation for $48 \mathrm{~h}$, invading and migrating cells on the bottom surface of each chamber were stained with Giemsa solution and photographed. The migrating cells were then counted in five random fields for quantification.

\section{Statistical analyses}

The continuous and categorical data are presented as mean \pm standard deviation (SD) and frequency (percentage), respectively. Comparisons of quantitative data between two and multiple groups were conducted by Student's t test and one-way analysis of variance (ANOVA), respectively, using GraphPad Prism 8.0. Kaplan-Meier curves with log-rank tests were used for univariate overall survival (OS) analysis. Cox regression models were used for multivariate OS analysis. Differences were considered to be statistically significant if $P<0.05$. 


\section{Results}

SOX17, SOX4, and VE-cadherin expression and VM are associated with clinical characteristics of ESCC

The positivity rates for SOX17 expression in the normal esophageal mucosa and ESCC samples were $83.3 \%$ and $41.4 \%$, respectively, and the difference was statistically significant $(P<0.05)$. There was no significant correlation between SOX17 protein expression and clinicopathological characteristics such as gender, age or tumor location ( $P>0.05$ ). SOX17 protein expression showed inverse correlations with tumor size, grade of differentiation, depth of invasion, and pathological tumor node metastasis (pTNM) stage. The rate of SOX17 expression was lower among cases with lymph node metastasis (LNM) than among those without LNM $(P<0.05)$. The rate of SOX4 protein expression in the normal esophageal mucosa was significantly higher than in ESCC ( $30 \%$ vs. $60 \%, P<0.05)$. The SOX4 expression rate showed positive correlations with the grade of differentiation, depth of infiltration, LNM, and pTNM stage of ESCC $(P<0.05)$. The rate of VE-cadherin protein expression in ESCC was significantly higher than in the normal esophageal mucosa (51.9\% vs. 3.3\%, $P<0.05)$. Moreover, VE-cadherin protein expression showed no correlation with patient age and gender. VE-cadherin protein expression was positively correlated with tumor size, grade of differentiation, LNM, pTNM stage, and depth of infiltration of ESCC $(P<0.05)$. The positive rate of VM in ESCC was 50\% (105/210), while no VM was found in the test group (Figure 1). VM positivity was not correlated with gender, age, tumor location, or histological grade $(P<0.05)$, but did correlate with tumor size, gross type, infiltration depth, LNM, and pTNM stage $(P<0.05)$. The above results are summarized in Table 1 and Fig. $1 \mathrm{~A}-\mathrm{H}$.

\section{Correlation analysis}

Spearman correlation analysis revealed that SOX17 expression in ESCC was negatively correlated with SOX4 expression ( $r=-0.418)$, VE-cadherin expression ( $r=0.487)$, and VM $(r=-0.609$, all $P<0.001$; Table 2$)$. On the other hand, SOX4 expression was positively correlated with VE-cadherin expression $(r=-0.576)$ and VM $(r=-0.447, P<0.001$; Table 2$)$. VE-cadherin expression also showed a positive correlation with VM $(r=-0.715, P<0.001 ;$ Table 2$)$.

\section{Survival analysis}

The 5-year OS rate in the ESCC group was 37.1\% (78/210). The OS of patients with SOX17 expression was significantly better than that of patients without SOX17 expression $(P<0.001$; Table 3, Figure $2 \mathrm{~A})$. On the other hand, the OS of patients with SOX4 expression, VE-cadherin expression, and VM was significantly lower than that of patients negative for these factors $(P<0.001$; Table 3 , Figure 2B-DI). 
On Cox regression model analysis, various factors such as gender, age, tumor type, tumor location, tumor diameter, histological grade, LNM, depth of invasion, pTNM stage, VM, SOX17 expression, SOX4 expression, and VE-cadherin expression were identified as prognostic factors. It was found that the expression of SOX17, SOX4, and VE-cadherin as well as VM were independent risk factors affecting the long-term prognosis of ESCC patients (Table 4).

Comparison of SOX17, SOX4, and VE-cadherin protein levels and mRNA levels in fresh ESCC and adjacent tissue samples

In fresh samples from 10 ESCC patients, the mean SOX17 protein $(0.816 \pm 0.337$ vs. $1.338 \pm 0.435, P<0.05)$ and mRNA $(0.223 \pm 0.373$ vs. $1.611 \pm 1.978, P<0.05)$ expression levels in ESCC tissues were significantly lower than the corresponding levels in the adjacent tissues ( $>5 \mathrm{~cm}$ away from the tumor). However, the mean SOX4 protein $(1.315 \pm 0.598$ vs. $0.554 \pm 0.393, P<0.05)$ and mRNA $(2.221 \pm 1.933$ vs. $0.402 \pm 0.403$, $P<0.05)$ expression levels in ESCC tissues were higher than the corresponding levels in the adjacent tissues. The mean VE-cadherin protein $(0.721 \pm 0.171$ vs. $0.494 \pm 0.175, P<0.05)$ and mRNA $(0.350 \pm 0.293$ vs. $0.092 \pm 0.071, P<0.05)$ expression levels were also significantly higher in ESCC tissues than in adjacent tissues. The results are shown in Figure 3.

Silencing VE-cadherin inhibits the invasion and migration of EC cells

Transwell experiments demonstrated that the migration and invasion abilities of the EC9706 and Eca109 cell lines were significantly lower in the VE-cadherin siRNA group than in the corresponding control groups $(P<0.05)$. No difference was observed between the control group and the control siRNA group (Figure 4A-D).

Additionally, the wound healing speed of cells in the VE-cadherin siRNA group was significantly slower than those in the corresponding control groups (Figure 4E-G).

Silencing VE-cadherin reduced EC cell proliferation

The proliferative abilities of EC9706 and Eca109 cells were significantly weakened after silencing of VEcadherin $(P<0.05$, Fig. $4 \mathrm{H})$. No significant difference was observed between the control group and the control siRNA group (Fig. 4H).

Silencing VE-cadherin increased SOX17 expression and decreased SOX4 expression 
After transfection, the VE-cadherin protein expression was significantly lower in the VE-cadherin siRNA group than in the control groups $(P<0.05)$. Moreover, SOX17 protein expression was significantly upregulated and SOX4 protein expression was downregulated in the VE-cadherin siRNA group (Fig. 5).

\section{Discussion}

The formation of VM can provide blood supply for the rapid proliferation of tumors, relieve the ischemic and hypoxic micro-environment around the tumors, and further accelerate the invasion and metastasis of tumors, which influences the clinical stage and long-term prognosis of cancer patients $[5,27]$. In this study, we confirmed the existence of VM in ESCC by IHC analysis of tumor tissues. At the same time, we found the VM was closely associated with the depth of invasion, pTNM stage, and LNM. The above findings are consistent with those of previous studies [28]. On survival analysis, the presence of VM was an independent poor prognostic factor in ESCC patients.

VE-cadherin, as an adhesion protein, can mediate the adhesion of cells to each other and maintain the further formation of tumor blood vessels. In the present study, VE-cadherin was found to be highly expressed in ESCC, and its positive expression was directly related to the depth of invasion, occurrence of LNM, and pTNM stage in ESCC. VE-cadherin expression was found to be an independent poor prognostic factor for ESCC patients. In vitro experiments suggested that high expression of VE-cadherin can accelerate ESCC invasion and metastasis, similar to the findings of previous reports [28]. Notably, after siRNA-mediated interference of bcl-2 expression in EC9706 cells under hypoxic conditions, the expression of VM-related molecules, such as VE-cadherin and matrix metalloproteinase 2 (MMP2), was significantly inhibited and VM generation was significantly reduced [29]. Moreover, VE-cadherin downregulation in melanoma is associated with the loss of VM formation [30]. Heinolainen et al. [31] and Han et al. [32] speculated that VE-cadherin might be an important determinant of VM in EC. Based on the findings of the present study, we firmly believe that VE-cadherin promotes the formation of VM in ESCC.

The SOX17 transcription factor has been known to have tumor suppressive function in ESCC [35-37]. SOX17 overexpression suppresses colony formation and cell migration/invasion in ESCC cell lines. In addition, SOX17 overexpression was found to inhibit tumor growth and metastasis in an ESCC xenograft model [33-35]. In the present study, we found significantly higher SOX17 expression in ESCC compared to the normal esophageal epithelium, confirming the tumor suppressive function of SOX17. A previous study demonstrated that hypermethylation of the promoter of the SOX17 gene leads to silencing of SOX17 protein expression in $\sim 50 \%$ ESCC patients [33]. In the present study, the low expression of SOX17 was significantly correlated with tumor differentiation, depth of invasion, LNM, and pTNM stage, suggesting that SOX17 acts as a tumor suppressor gene in ESCC. Moreover, SOX17 expression was an independent predictor of prognosis in this study. 
The present study found that SOX4 protein and mRNA expression levels were significantly increased in ESCC. Moreover, the high expression of SOX4 promoted the invasion of ESCC. Schilham et al. also found that SOX4 overexpression is closely associated with ESCC metastasis [36]. SOX4 has been found to be involved in tumorigenesis and tumor invasion in different types of cancers through the activated Wnt, transforming growth factor (TGF)- $\beta$, Hedgehog and Notch pathways or the regulation of microRNA expression [30]. Zilong et al. speculated that miR-212-3p targets SOX4 to inhibit the Wnt/ $\beta$-catenin signaling pathway and promote apoptosis in ESCC [37].

VM is closely related to tumor growth, invasion, metastasis, and long-term prognosis of cancer patients [38-40]. In the present study, we confirmed that VM formation in ESCC was positively correlated with high SOX4 expression and low SOX17 expression. These findings indicate that VE-cadherin may promote the formation of VM in ESCC by affecting the expression levels of SOX4 and SOX17. In a previous study, we confirmed that SOX4 may promote the formation of VM by promoting EMT in ESCC [23]. Studies have shown that in a hypoxic environment, hypoxia-inducible factor (HIF)-2a, a VM-initiating factor, is activated, which increases VE-cadherin transcription. VE-cadherin, in turn, induces repositioning of EphA2 to the cell membrane. Furthermore, PI3K is activated by VE-cadherin and EphA2 simultaneously. The activated PI3K regulates the activation of the pre-gene of membrane type 1 matrix metalloproteinase (MT1-MMP). The combination of MT1-MMP and MMP2 promotes the fragmentation of laminin 5y25y2 $(\mathrm{LN}-5 \mathrm{\gamma} 2)$ chains into fragments $(5 \gamma 2$ and $5 \gamma 2 \chi)$, and increased levels of these two fragments in the extracellular micro-environment eventually leads to the formation of a VM net-like structure [41].

In conclusion, the present study found low SOX17 expression, high SOX4 expression, and high VEcadherin expression in ESCC. Moreover, the expression of these proteins was closely associated with VM in ESCC. We believe that development of targeted therapies to suppress SOX4 expression or enhance SOX17 expression may impair the formation of VM, thereby prolonging the survival of ESCC patients. Future studies are required to determine the exact pathophysiological mechanism linking SOX4, SOX17, VE-cadherin, and VM.

\section{Abbreviations}

ESCC: esophageal squamous cell carcinoma; VM: vasculogenic mimicry; EMT: epithelial-mesenchymal transition; EC: esophageal cancer; LNM: lymph node metastasis; PTNM: pathology tumor node metastasis; VE-cadherin: vascular endothelial cadherin; SRY: sex determining region on Y; HMG box: high mobility group box; SOX17: Sex determining region of Y chromosome box 17; SOX4: Sex determining region of $Y$ chromosome box 4; PBS: phosphate-buffered saline; AVONA: one-way analysis of variance; OS: overall survival; FAK: focal adhesion kinase; ERK1/2: extracelluar signal regulated kinase 1 and 2; 
DAB: 3,3-N-diaminobenzidine tetrahydrochloride; FBS: fetal bovine serum; RIPA: radioimmunoprecipitation assay; PMFS: phenylmethylsulfonyl fluoride; SDS-PAGE: sodium dodecyl sulfatepolyacrylamide gel electrophoresis; PVDF: polyvinylidene fluoride.

\section{Declarations}

Author contributions: Yanzi Qin, Wenjun Zhao and Zhaogeng Cai contributed equally to this work. Yanzi Qin and Li Ma participated in the research design. Yanzi Qin, Wenjun Zhao, Qi Wang, Jin Gao, Ningning Yang and Hongfei Ci performed the research and wrote the manuscript. Zhaogeng Cai, Li Ma and Zhenzhong Feng contributed towards critically revising the manuscript.

Funding information: This work was funded by the Natural Science Key Project of Anhui Provincial Department of Education (KJ2019A0344), the 512 Talent Cultivation Plan of Bengbu Medical College (by51201305), and the Natural Science Key Project of Bengbu Medical College (BYKY1817ZD).

Competing interests: The authors declare that they have no competing interests.

Ethical approval: This experiment was approved by the Ethics Committee of Bengbu Medical College (No. 055). This study was performed in accordance with the ethical standards put forth in the 1964 Declaration of Helsinki.

\section{References}

[1] Bray F, Ferlay J, Soerjomataram I, Siegel RL, Torre LA, Jemal A. Global cancer statistics 2018: GLOBOCAN estimates of incidence and mortality worldwide for 36 cancers in 185 countries. CA Cancer $J$ Clin. 2018; 68(6): 394-424.

[2] Chen W, Zheng R, Baade PD, Zhang S, Zeng H, Bray F, Jemal A, Yu XQ, He J. Cancer statistics in China, 2015. CA Cancer J Clin. 2016; 66(2): 115-32.

[3] Ajani JA, D'Amico TA, Almhanna K, Bentrem DJ, Besh S, Chao J, Das P, Denlinger C, Fanta P, Fuchs CS, Gerdes H, Glasgow RE, Hayman JA, Hochwald S, Hofstetter WL, Ilson DH, Jaroszewski D, Jasperson K, Keswani RN, Kleinberg LR, Korn WM, Leong S, Lockhart AC, Mulcahy MF, Orringer MB, Posey JA, Poultsides GA, Sasson AR, Scott WJ, Strong VE, Varghese TK Jr, Washington MK, Willett CG, Wright CD, Zelman D, McMillian N, Sundar H; National comprehensive cancer network. Esophageal and esophagogastric junction cancers, version1. 2015. J Natl Compr Canc Netw. 2015; 13(2):194-227. 
[4] Delgado-Bellido D, Fernández-Cortés M, Rodríguez MI, Serrano-Sáenz S, Carracedo A, Garcia-Diaz A, Oliver FJ. VE-cadherin promotes vasculogenic mimicry by modulating kaiso-dependent gene expression. Cell Death Differ. 2019; 26(2):348-61.

[5] Zhang J, Zhang G, Hu P, Deng G, Liu Q, Qiao L, Luo H, Zhang J. Vasculogenic mimicry is associated with increased tumor-infiltrating neutrophil and poor outcome in esophageal squamous cell carcinoma. Onco Targets Ther. 2017; 10: 2923-30.

[6] Cappelli HC, Kanugula AK, Adapala RK, Amin V, Sharma P, Midha P, Paruchuri S, Thodeti CK. Mechanosensitive TRPV4 channels stabilize VE-cadherin junctions to regulate tumor vascular integrity and metastasis. Cancer Lett. 2019; 442: 15-20.

[7] Paatero I, Sauteur L, Lee M. Junction-based lamellipodia drive endothelial cell rearrangements in vivo via a VE-cadherin-F-actin based oscillatory cell-cell interaction. Nat Commun, 2018, (10): 3545

[8] Yeo C, Lee HJ, Lee EO. Serum promotes vasculogenic mimicry through the EphA2/VE-cadherin/AKT pathway in PC-3 human prostate cancer cells. Life Sci, 2019, 221: 267-73.

[9] Delgado-Bellido D, Fernández-Cortés M, Rodríguez MI, Serrano-Sáenz S, Carracedo A, Garcia-Diaz A, Oliver FJ. VE-cadherin promotes vasculogenic mimicry by modulating kaiso-dependent gene expression. Cell Death Differ. 2019; 26(2): 348-61.

[10] Gubbay J, Collignon J, Koopman P, Capel B, Economou A, Münsterberg A, Vivian N, Goodfellow P, Lovell-Badge R. A gene mapping to the sex-determining region of the mouse $Y$ chromosome is a member of a novel family of embryonically expressed genes. Nature. 1990; 346(6281): 245-50.

[11] Lovell-Badge R. The early history of the Sox genes [J]. Int J Biochem Cell Biol. 2010; 42(3): 378-80.

[12] Hou L, Srivastava Y, Jauch R. Molecular basis for the genome engagement by Sox proteins. Semin Cell Dev Biol. 2017; 63: 2-12.

[13] Kamachi Y, Kondoh $\mathrm{H}$. Sox proteins: regulators of cell fate specification and differentiation. Development. 2013; 140(20): 4129-44.

[14] Lefebvre V, Dumitriu B, Penzo-Méndez A, Han Y, Pallavi B. Control of cell fate and differentiation by Sry-related high-mobility-group box (Sox) transcription factors. Int J Biochem Cell Biol. 2007; 39(12): 2195-214.

[15] Kanai Y, Kanai-Azuma M, Noce T, Saido TC, Shiroishi T, Hayashi Y, Yazaki K. Identification of two Sox17 messenger RNA isoforms, with and without the high mobility group box region, and their differential expression in mouse spermatogenesis. J Cell Biol. 1996; 133(3): 667-81.

[16] Zhang Y, Bao W, Wang K, Lu W, Wang H, Tong H, Wan X. SOX17 is a tumor suppressor in endometrial cancer. Oncotarget. 2016; 7(46): 76036-46. 
[17] Sinner D, Kordich JJ, Spence JR, Opoka R, Rankin S, Lin SC, Jonatan D, Zorn AM, Wells JM. Sox17 and Sox4 differentially regulate beta-catenin/T-cell factor activity and proliferation of colon carcinoma cells. Mol Cell Biol. 2007; 27(22): 7802-15.

[18] Zhang W, Glöckner SC, Guo M, Machida EO, Wang DH, Easwaran H, Van Neste L, Herman JG, Schuebel KE, Watkins DN, Ahuja N, Baylin SB. Epigenetic inactivation of the canonical Wnt antagonist SRY-box containing gene 17 in colorectal cancer. Cancer Res. 2008; 68(8): 2764-72.

[19] Yin D, Jia Y, Yu Y, Brock MV, Herman JG, Han C, Su X, Liu Y, Guo M. SoX17 methylation inhibits its antagonism of Wnt signaling pathway in lung cancer. Discov Med. 2012; 14(74): 33-40.

[20] Jia Y, Yang Y, Liu S, Herman JG, Lu F, Guo M. SOX17 antagonizes WNT/ $\beta$-catenin signaling pathway in hepatocellular carcinoma. Epigenetics. 2010; 5(8): 743-9.

[21] Moreno CS. The Sex-determining region Y-box 4 and homeobox C6 transcriptional networks in prostate cancer progression: crosstalk with the Wnt, Notch, and PI3K pathways. Am J Pathol. 2010; 176(2): 518-27.

[22] Rhodes DR, Yu J, Shanker K, Deshpande N, Varambally R, Ghosh D, Barrette T, Pandey A, Chinnaiyan AM. Large-scale meta-analysis of cancer microarray data identifies common transcriptional profiles of neoplastic transformation and progression. Proc Natl Acad Sci USA. 2004; 101(25): 9309-14.

[23] Aaboe M, Birkenkamp-Demtroder K, Wiuf C, Sørensen FB, Thykjaer T, Sauter G, Jensen KM, Dyrskjøt L, Ørntoft T. SOX4 expression in bladder carcinoma: clinical aspects and in vitro functional characterization. Cancer Res. 2006; 66(7): 3434-42.

[24] Qin Y, Zhao W, Cheng L, Wu S, Wu Q, Gao J, Bian Z, Ma L. Clinical significance of vasculogenic mimicry, vascular endothelial cadherin and SOX4 in patients with esophageal squamous cell carcinoma. Int J Clin Exp Pathol. 2019; 12(7): 2462-73.

[25] Xia J, Cheng L, Mei C, Ma J, Shi Y, Zeng F, Wang Z, Wang Z. Genistein inhibits cell growth and invasion through regulation of miR-27a in pancreatic cancer cells. Curr Pharm Des. 2014; 20(33): 534853.

[26] Yang Q, Huang J, Wu Q, Cai Y, Zhu L, Lu X, Chen S, Chen C, Wang Z. Acquisition of epithelialmesenchymal transition is associated with Skp2 expression in paclitaxel-resistant breast cancer cells. $\mathrm{Br}$ J Cancer. 2014; 110(8): 1958-67.

[27] Tang NN, Zhu H, Zhang HJ, Zhang WF, Jin HL, Wang L, Wang P, He GJ, Hao B, Shi RH. HIF-1a induces VE-cadherin expression and modulates vasculogenic mimicry in esophageal carcinoma cells. World $\mathrm{J}$ Gastroenterol. 2014; 20(47): 17894-904.

[28] Cappelli HC, Kanugula AK, Adapala RK, Amin V, Sharma P, Midha P, Paruchuri S, Thodeti CK. Mechanosensitive TRPV4 channels stabilize VE-cadherin junctions to regulate tumor vascular integrity 
and metastasis. Cancer Lett. 2019; 442: 15-20.

[29] Zhang J, Qiao L, Liang N, Xie J, Luo H, Deng G, Zhang J. Vasculogenic mimicry and tumor metastasis. J BUON. 2016; 21(3): 533-41.

[30] Hendrix MJ, Seftor EA, Meltzer PS, Gardner LM, Hess AR, Kirschmann DA, Schatteman GC, Seftor RE. Expression and functional significance of VE-cadherin in aggressive human melanoma cells: role in vasculogenic mimicry. Proc Natl Acad Sci USA. 2001; 98(14): 8018-23.

[31] Heinolainen K, Karaman S, D'Amico G, Tammela T, Sormunen R, Eklund L, Alitalo K, Zarkada G. VEGFR3 Modulates Vascular Permeability by Controlling VEGF/VEGFR2 Signaling. Circ Res. 2017; 120(9): 1414-25.

[32] Han H, Du L, Cao Z, Zhang B, Zhou Q. Triptonide potently suppresses pancreatic cancer cell-mediated vasculogenic mimicry by inhibiting expression of VE-cadherin and chemokine ligand 2 genes. Eur $\mathrm{J}$ Pharmacol. 2018; 818: 593-603.

[33] Kuo IY, Wu CC, Chang JM, Huang YL, Lin CH, Yan JJ, Sheu BS, Lu PJ, Chang WL, Lai WW, Wang YC. Low SOX17 expression is a prognostic factor and drives transcriptional dysregulation and esophageal cancer progression. Int J Cancer. 2014; 135(3): 563-73.

[34] Zhang W, Glöckner SC, Guo M, Machida EO, Wang DH, Easwaran H, Van Neste L, Herman JG, Schuebel KE, Watkins DN, Ahuja N, Baylin SB. Epigenetic inactivation of the canonical Wnt antagonist SRY-box containing gene 17 in colorectal cancer. Cancer Res. 2008; 68(8): 2764-72.

[35] Kuo IY, Huang YL, Lin CY, Lin CH, Chang WL, Lai WW, Wang YC. SOX17 overexpression sensitizes chemoradiation response in esophageal cancer by transcriptional down-regulation of DNA repair and damage response genes. J Biomed Sci. 2019; 26(1): 20.

[36] Schilham MW, Oosterwegel MA, Moerer P, Ya J, de Boer PA, van de Wetering M, Verbeek S, Lamers WH, Kruisbeek AM, Cumano A, Clevers H. Defects in cardiac outflow tract formation and pro-Blymphocyte expansion in mice lacking Sox-4. Nature. 1996; 380(6576): 711-4.

[37] Wu Z, Yu B, Jiang L. MiR-212-3p mediates apoptosis and invasion of esophageal squamous cell carcinoma through inhibition of the Wnt/ $\beta$-catenin signaling pathway by targeting SOX4. J Thorac Dis. 2020; 12(8):4357-67.

[38] Hulin JA, Tommasi S, Elliot D, Mangoni AA. Small molecule inhibition of DDAH1 significantly attenuates triple negative breast cancer cell vasculogenic mimicry in vitro [J]. Biomed Pharmacother, 2019, (111): 602-612.

[39] Bai J, Yeh S, Qiu X, Hu L, Zeng J, Cai Y, Zuo L, Li G, Yang G, Chang C. TR4 nuclear receptor promotes clear cell renal cell carcinoma (ccRCC) vasculogenic mimicry (VM) formation and metastasis via altering the miR490-3p/vimentin signals. Oncogene. 2018; 37(44): 5901-12. 
[40] Kawahara R, Niwa Y, Simizu S. Integrin $\beta 1$ is an essential factor in vasculogenic mimicry of human cancer cells. Cancer Sci. 2018; 109(8): 2490-6.

[41] Ge H, Luo H. Overview of advances in vasculogenic mimicry - a potential target for tumor therapy. Cancer Manag Res. 2018; 10: 2429-37.

\section{Figures}

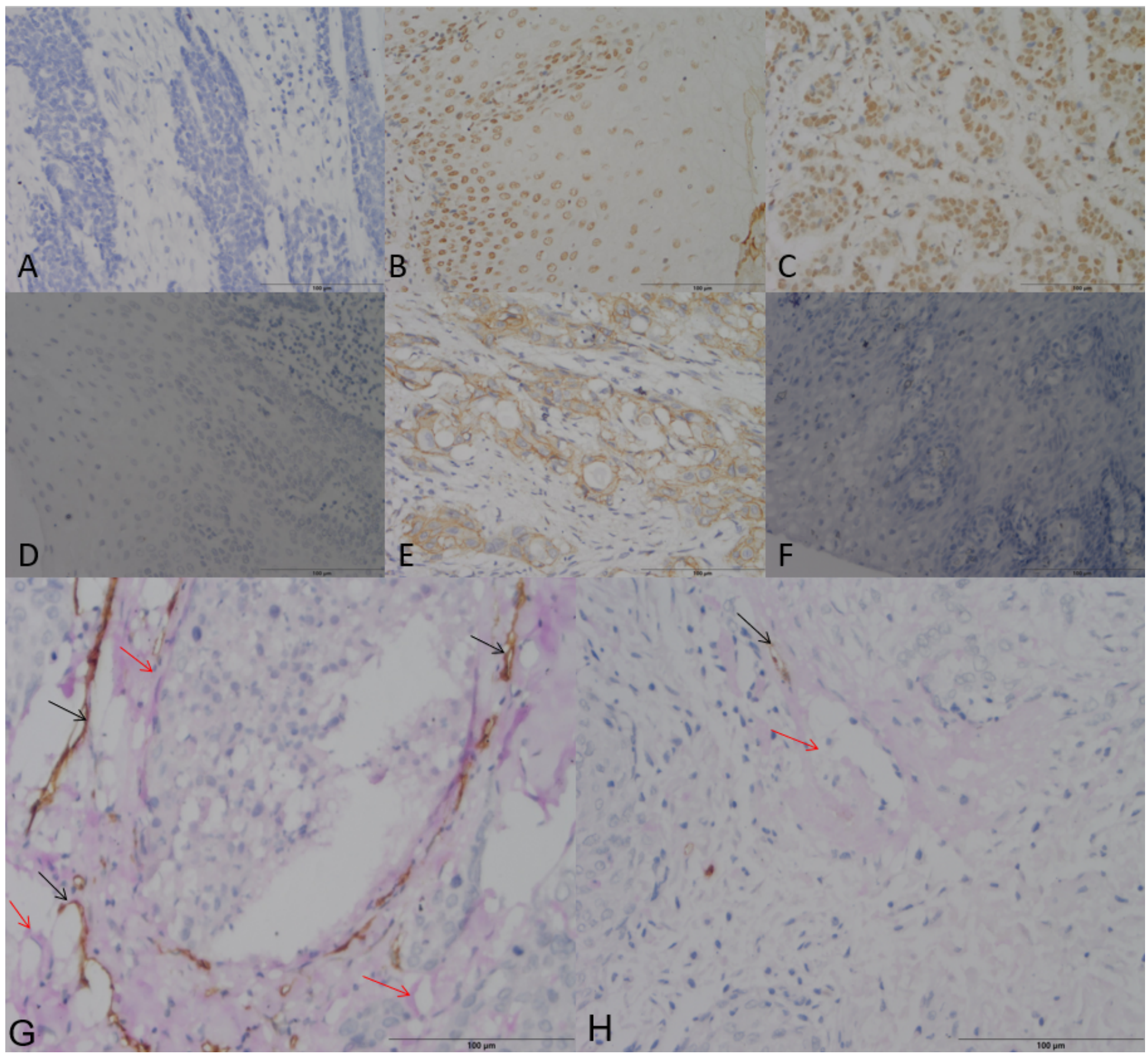

\section{Figure 1}


Immunohistochemical analysis of SOX17, SOX4, and VE-cadherin expression as well as VM in ESCC and normal esophageal tissues ( $\times 400$ magnification): negative staining of SOX17 in ESCC (A), positive staining of SOX17 in the nucleus of normal esophageal tissue (B), positive staining of SOX4 in the nucleus of ESCC (C), negative staining of SOX4 in the normal esophageal tissue (D), positive staining of VE-cadherin in the cell membrane and plasma of ESCC (E), negative staining of VE-cadherin in the normal esophageal tissue (F), VM in ESCC (G, red arrow), positive staining of normal blood vessels with CD34 (H, black arrow).
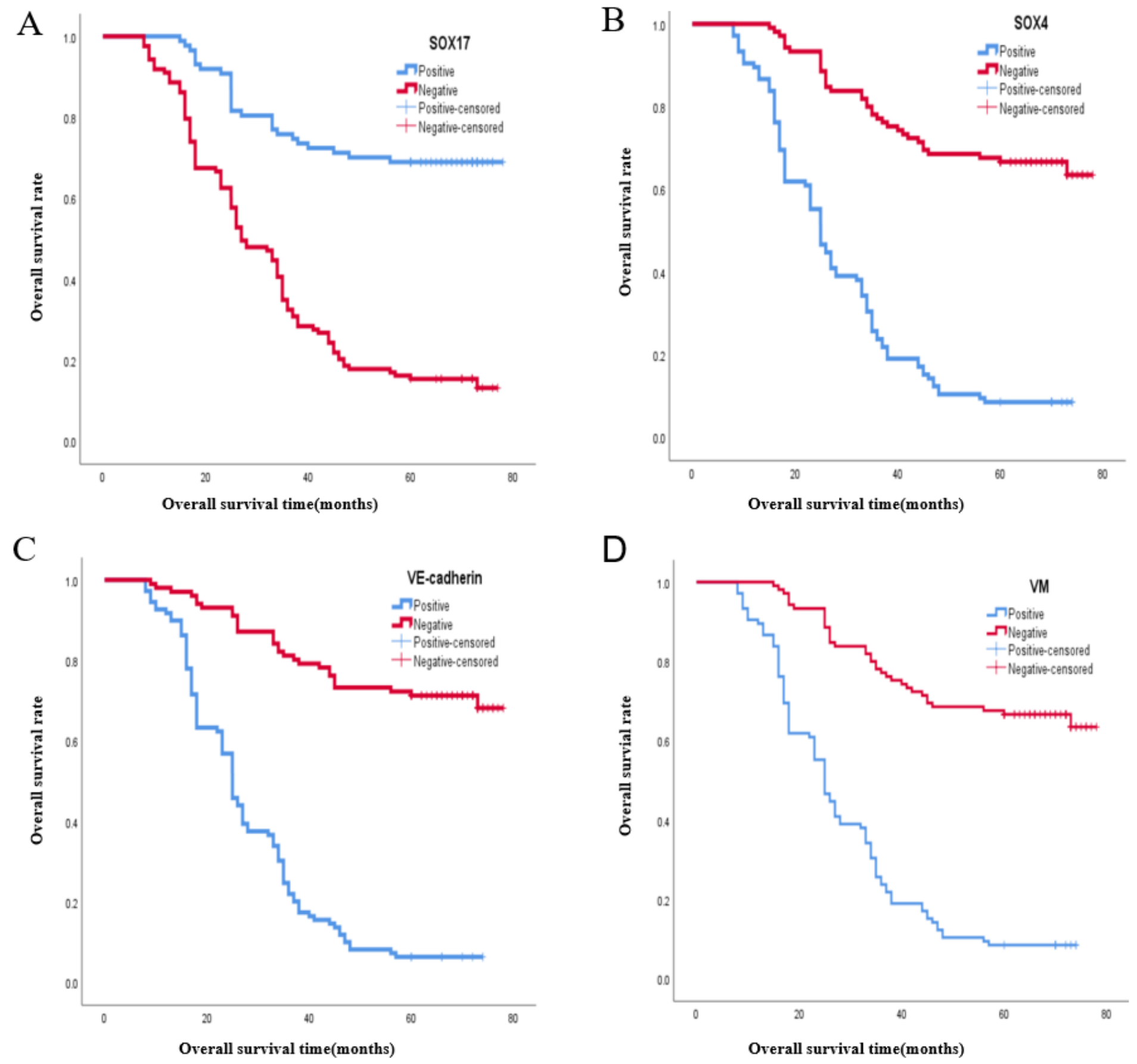

Figure 2 
Kaplan-Meier analysis of the survival rates of patients with ESCC: higher overall survival of patients with positive SOX17 expression (I, log-rank $=61.200, P<0.001)$, lower overall survival of patients with positive SOX4 expression $(\mathrm{J}$, log-rank $=70.109, \mathrm{P}<0.001)$, overall survival of patients in relation to VE-cadherin $(\mathrm{K}$, log-rank $=32.174, P<0.001)$, lower overall survival of patients with presence of $V M(L$, log-rank $=92.811$, $\mathrm{P}<0.001)$. Blue lines: cases positive for SOX17, and SOX4, VE-cadherin expression and VM; red line: cases negative for SOX17, SOX4, and VE-cadherin expression and VM.

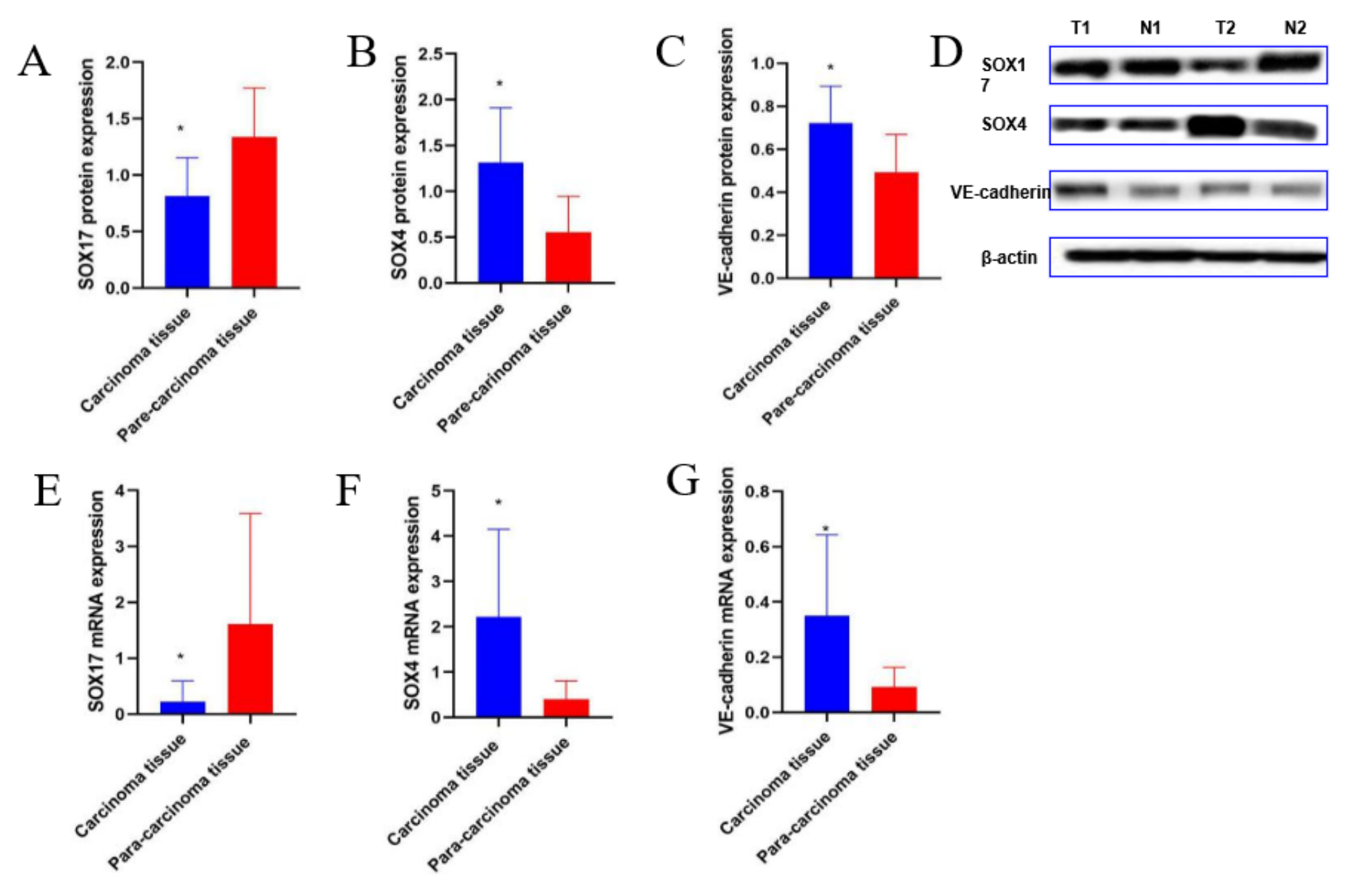

Figure 3

SOX17, SOX4, and VE-cadherin protein and mRNA expression. A, B, C. Graphical representation of SOX17, SOX4, and VE-cadherin protein expression, respectively ( $\left.{ }^{*} P<0.05\right)$. D. Western blot analysis of SOX17, SOX4, and VE-cadherin protein levels in ESCC tissues and non-tumor tissues ( $T 1$, T2 correspond to ESCC tissues; N1, N2 correspond to normal mucosal tissues). E, F, G. Graphical representation of SOX17, SOX4, and VE-cadherin mRNA expression, respectively $(* P<0.05)$. 

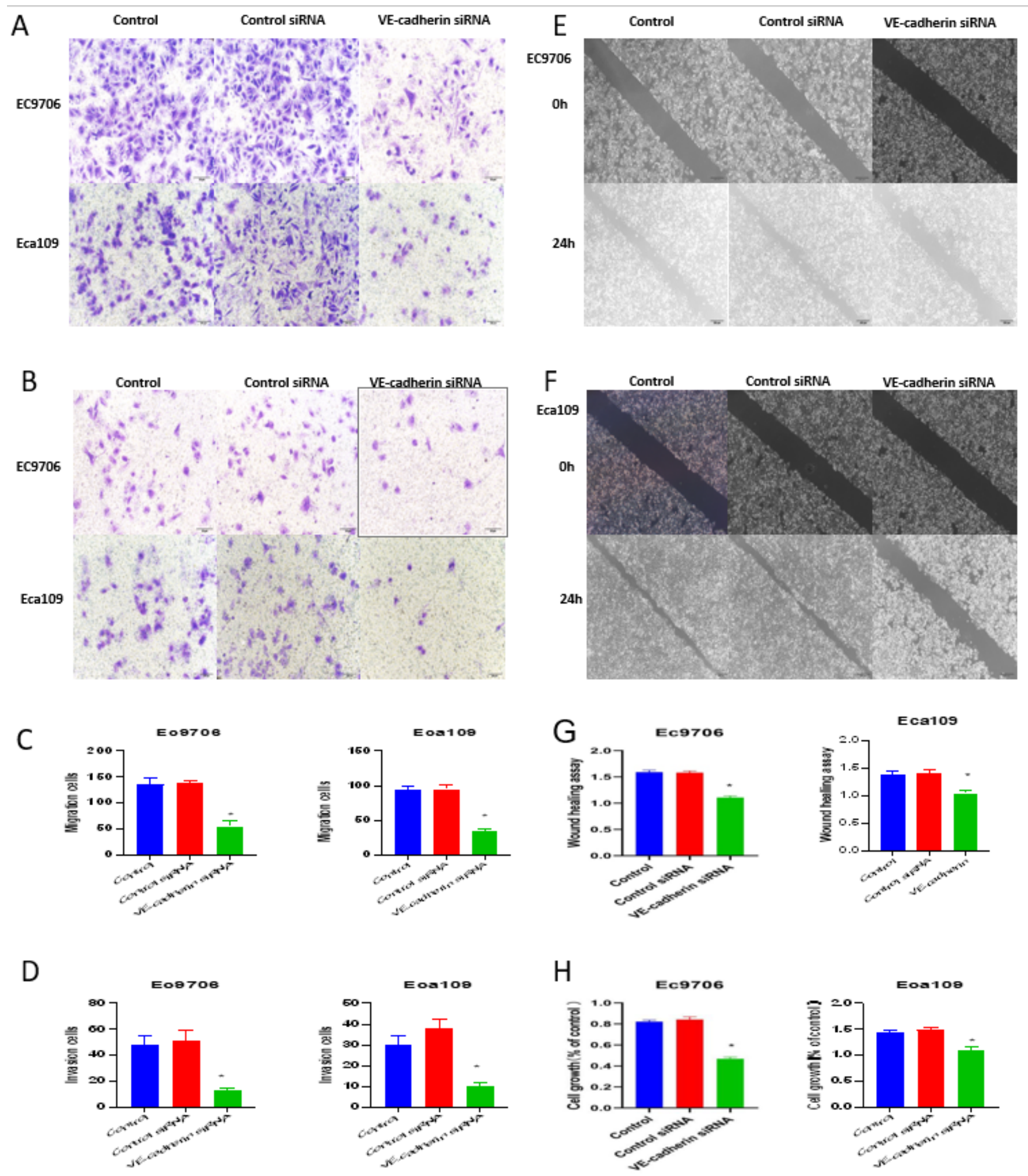

\section{Figure 4}

A D. Migration and invasion abilities of EC cells after VE-cadherin silencing. Transwell assay showing the migration of the EC9706 and Eca109 cells (A); Transwell assay showing the invasion of EC9706 and Eca109 cells (B); Graphical representation of migration and invasion among EC9706 and Eca109 cells (C, $D)$. The wound healing experiment showed the effect of silencing VE-cadherin on the migration and 
healing ability of EC9706 and Eca109 cells (E, F, G). Comparison of in vitro proliferation of EC9706 and Eca109 cells $(H) .{ }^{*} \mathrm{P}<0.05$ vs control or control siRNA.

\section{A EC9706}
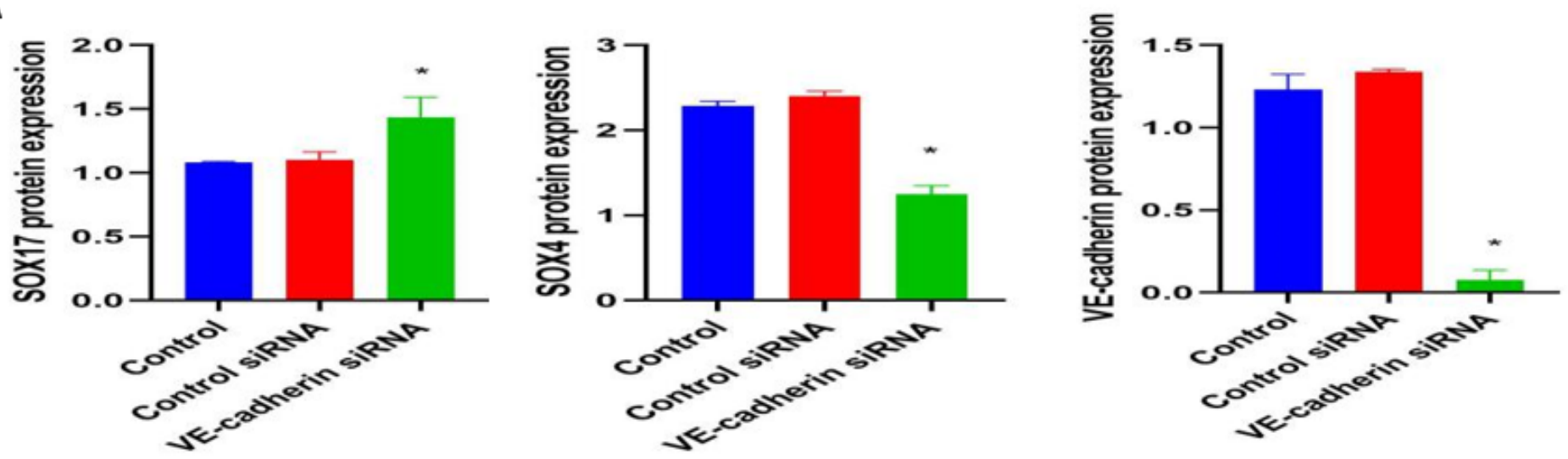

B Eca109
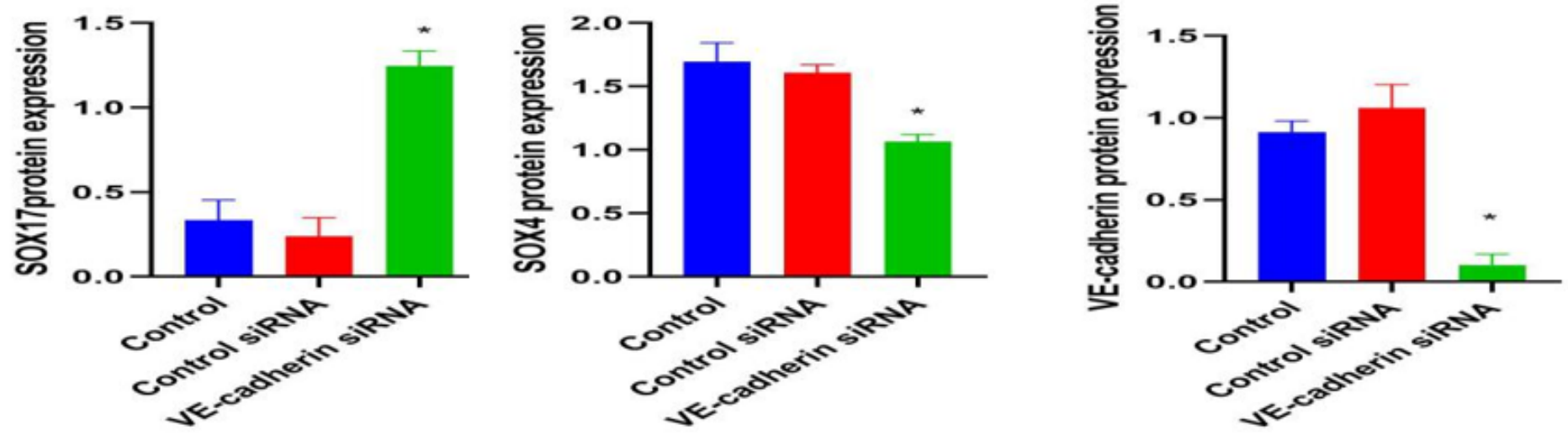

C

VE-cadherin

SOX17

SOX4

$\beta$-actin
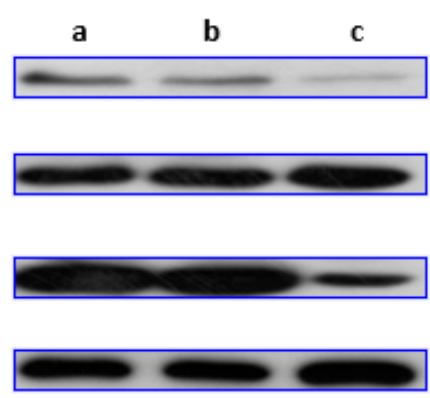

EC9706

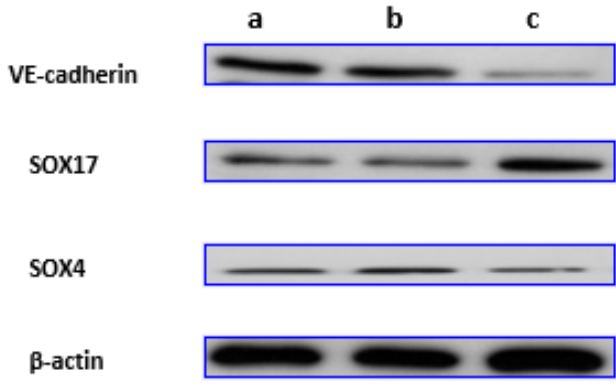

Eca109

Figure 5

Top panel: Western blot analysis to detect the expression of SOX17, SOX4 and VE-cadherin in the three groups of EC9706 and Eca109 cells after VE-cadherin silencing (A, B). Quantitative western blot data (C, $D)$. (a indicates the control group; $b$ indicates the control siRNA group; and $c$ indicates the VE-cadherin siRNA group). ${ }^{*} \mathrm{P}<0.05$ vs control or control siRNA 